\title{
Two Alternative Methods to Measure the Radiated Emission in a Reverberation Chamber
}

\author{
Qian Xu, ${ }^{1}$ Yi Huang, ${ }^{1}$ Sheng Yuan, ${ }^{1}$ Lei Xing, ${ }^{2}$ and Zhihao Tian ${ }^{1}$ \\ ${ }^{1}$ Department of Electrical Engineering and Electronics, The University of Liverpool, Liverpool, L69 3GJ, UK \\ ${ }^{2}$ College of Electronic and Information Engineering, Nanjing University of Aeronautics and Astronautics, Nanjing 211106, China
}

Correspondence should be addressed to Yi Huang; yi.huang@liverpool.ac.uk

Received 21 December 2015; Accepted 8 February 2016

Academic Editor: Jaume Anguera

Copyright (C) 2016 Qian Xu et al. This is an open access article distributed under the Creative Commons Attribution License, which permits unrestricted use, distribution, and reproduction in any medium, provided the original work is properly cited.

One of the most important applications of a reverberation chamber is for radiated power measurement. Two new alternative methods are proposed to measure the radiated power of equipment under test in a reverberation chamber in this paper. Traditionally, as stated in IEC 61000-4-21 standard, this measurement requires two antennas, a signal generator and a spectrum analyzer or receiver. However, it is found that if a signal generator is not available, a vector network analyzer can be used to complete the measurement. Furthermore, if only one antenna is available, by taking advantage of the enhanced backscattering effect, the measurement can still be completed. By incorporating the one- and/or two-antenna methods, the radiation efficiency of antennas is also considered. Measurements have been conducted to verify the proposed methods, the results from these three methods are compared, and a very good agreement is obtained. It is found that the two proposed methods are simpler than the conventional method.

\section{Introduction}

New measurement methods in the reverberation chamber (RC) have been developed in recent years such as antenna measurements [1-7], material characterization [8-11], shielding effectiveness measurements [12-16], and channel emulation [17-25]. An RC has been used to measure the radiated emission (RE) of equipment under test (EUT) since 1976 [26]. The standard procedure has been well established in IEC 6100-4-21 standard [27]. In 2006, efforts were made to simplify the measurement procedure; by measuring the received power in the time domain, the RC does not need to be calibrated in advance $[28,29]$. However, this method requires a signal generator (SG) to be switched off quickly (much smaller than the chamber decay time) and a spectrum analyzer (SA) needs to work in a zero-span mode to record the received power in the time domain. Moreover, the SG and the SA must be synchronized for trigger. These could be a problem for some instruments. In 2011, new methods on antenna radiation efficiency measurement without reference antenna in RC were developed (the one-, two-, and three-antenna method) [30]. This offers an opportunity to further simplify the RE measurement procedure in an RC.

In this paper, two new alternative methods are proposed by incorporating the one- and/or two-antenna methods into the RE measurement. The method in the IEC standard requires SG and SA, while the proposed methods require a vector network analyzer (VNA) and SA. The time domain response of the RC is extracted from the inverse fast Fourier transform (IFFT) of the collected $S$-parameters, which normally gives better dynamic range than directly measuring it in the time domain $[28,29]$ and the radiation efficiency of antennas could be measured at the same time. It is interesting to note that, by taking advantage of the enhanced backscattering effect [31], only one antenna is required. Both proposed methods do not need the RC to be calibrated in advance.

In Section 2, the method in the IEC standard is reviewed first and followed by the two proposed methods. Measurements are conducted to verify the results by using three methods (the method in IEC standard and two proposed methods) in Section 3. And finally, discussions and conclusions are given in Section 4. 


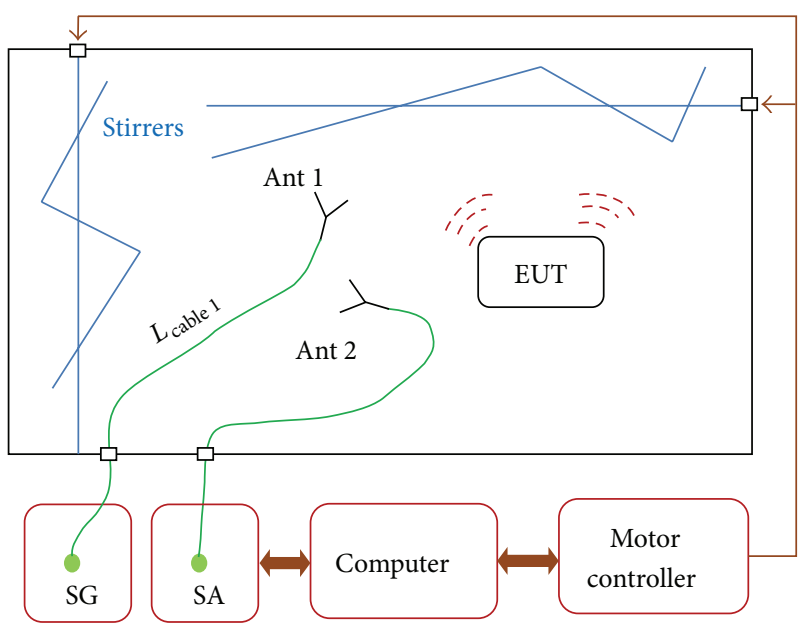

FIgURE 1: The RE measurement system in the IEC standard.

\section{RE Measurement Methods in RC}

2.1. Method in the IEC Standard. The method in the IEC standard [27] is reviewed first; either averaged or maximum received power can be employed. The averaged received power is used in this paper, because it has a lower uncertainty [27]. The measurement system is shown in Figure 1 where the computer controls the operation of the stirrers and records the reading from the SA for each stirrer position over the frequency band of interest. In this paper the stirrers rotate step by step (in mode-tuned operation [27]).

The measurement procedure is given as follows:

(1) The EUT is turned on; the SG is replaced with a $50 \mathrm{Ohm}$ load. Then record the SA reading for each stirrer position.

(2) Turn off the EUT, connect antenna 1 to the SG (reference source), and record the SA reading for each stirrer position. from

The radiated power of the EUT $\left(P_{\text {radEUT }}\right)$ can be obtained

$$
\begin{aligned}
P_{\text {radEUT }} & =\frac{\left\langle P_{\text {SAEUT }}\right\rangle}{\left\langle P_{\text {SAref }}\right\rangle} P_{\text {rad } 1}, \\
P_{\text {rad 1 }} & =P_{\text {ref }} L_{\text {cable } 1}\left(1-\left|S_{11}\right|^{2}\right) \eta_{1},
\end{aligned}
$$

where $\left\langle P_{\text {SAEUT }}\right\rangle$ is the averaged received power (SA readings) from the EUT for all stirrer positions, $\left\langle P_{\mathrm{SAref}}\right\rangle$ is the averaged received power (SA readings) from the reference source for all stirrer positions, $P_{\operatorname{rad} 1}$ is the radiated power of antenna 1 when connected to the SG, $P_{\text {ref }}$ is the output power from SG, $L_{\text {cable } 1}$ is the loss of the cable between antenna 1 and SG, $S_{11}$ is the reflection coefficient of antenna 1 , and $\eta_{1}$ is the radiation efficiency of antenna 1.

Two issues should be noted when following the procedure in the IEC standard [27]:

(1) The loss of the cable must be known.

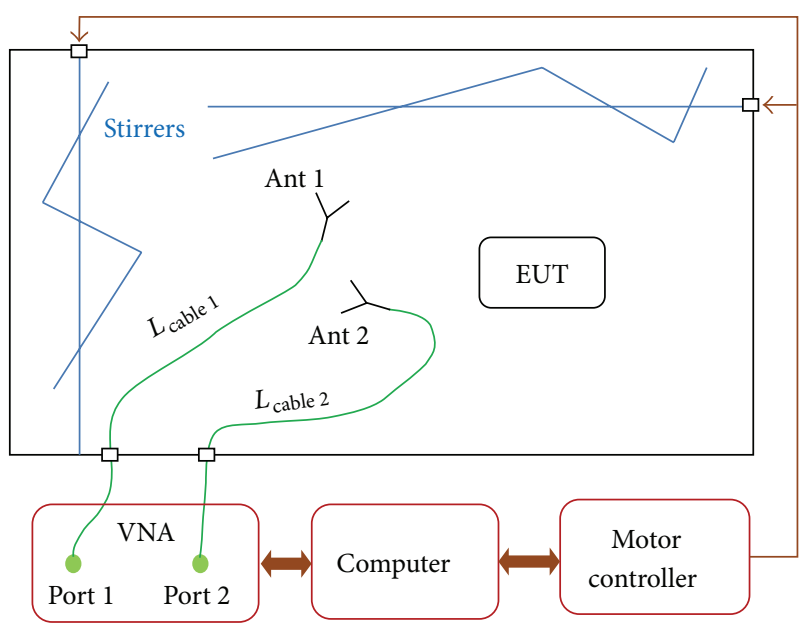

FIGURE 2: The RE measurement system with VNA and two antennas.

(2) The performance of antenna 1 must be known $\left(S_{11}\right.$ and $\left.\eta_{1}\right)$.

Both of these measurements need VNA: for the radiation efficiency measurement, the IEC standard also gives a standard procedure (which needs a reference antenna with known radiation efficiency), but a separate measurement needs to be conducted to characterize the performance of the antenna which is time consuming.

However, by combing the one- and two-antenna methods in [30], this extra measurement is not necessary as we will see from the proposed methods below.

2.2. Method Using VNA and Two Antennas. The first measurement step is the same as Step 1 in the IEC standard. For Step 2, as shown in Figure 2, the EUT is turned off, and we use a VNA and connect port 1 to antenna 1 and port 2 to antenna 2 , respectively. The reference plane of the VNA is calibrated at the ends of cable 1 and cable 2 (at the input port of the antennas).

By applying the chamber transfer function between antenna 1 and antenna 2, we have

$$
T_{21}=\frac{\left\langle\left|S_{21, s}\right|^{2}\right\rangle}{\left(1-\left|\left\langle S_{22}\right\rangle\right|^{2}\right)\left(1-\left|\left\langle S_{11}\right\rangle\right|^{2}\right) \eta_{1} \eta_{2}},
$$

where $S_{21, s}$ is the stirred part of $S$-parameters:

$$
S_{21, s}=S_{21}-\left\langle S_{21}\right\rangle
$$

$\langle\cdot\rangle$ means the averaged value of $S$-parameter using any stirring method (mechanical stir, frequency stir, source stir, etc.) and $\eta_{1}$ and $\eta_{2}$ are the radiation efficiency of antenna 1 and antenna 2 , respectively.

Note that the measurement setup in Figure 2 is the same as in the two-antenna measurement method (the EUT can be 
regarded as a load), and the radiation efficiency of antenna 1 and antenna 2 can be obtained using [30]

$$
\eta_{i}=\frac{1}{\left(1-\left|\left\langle S_{i i}\right\rangle\right|^{2}\right)} \sqrt{\frac{C_{\mathrm{RC}}}{\omega e_{b} \tau_{\mathrm{RC}}}\left\langle\left|S_{i i, s}\right|^{2}\right\rangle} \quad(i=1 \text { or } 2),
$$

where $C_{\mathrm{RC}}$ is the chamber constant, $C_{\mathrm{RC}}=16 \pi^{2} V / \lambda^{3}, V$ is the volume of the chamber, $\lambda$ is the free-space wavelength, $\omega=2 \pi f$ is the angular frequency, $\tau_{\mathrm{RC}}$ is the chamber decay time which can be obtained from the IFFT of $S$-parameters [22], and $e_{b}$ is the enhanced backscatter constant [30,31]:

$$
e_{b}=\frac{\sqrt{\left\langle\left|S_{11, s}\right|^{2}\right\rangle\left\langle\left|S_{22, s}\right|^{2}\right\rangle}}{\left\langle\left|S_{21, s}\right|^{2}\right\rangle} .
$$

By applying the chamber transfer function between antenna 2 and the EUT, we have

$$
T_{2 \text { EUT }}=\frac{\left\langle P_{\text {SAEUT }}\right\rangle}{P_{\text {radeUt }}} \frac{1}{L_{\text {cable } 2}\left(1-\left|\left\langle S_{22}\right\rangle\right|^{2}\right) \eta_{2}} .
$$

If the chamber is well-stirred, $T_{21}=T_{2 \mathrm{EUT}}$, and the radiated power from the EUT ( $\left.P_{\text {radeUT }}\right)$ can be solved as

$$
P_{\text {radeUt }}=\frac{\left\langle P_{\text {SAEUT }}\right\rangle\left(1-\left|\left\langle S_{11}\right\rangle\right|^{2}\right) \eta_{1}}{L_{\text {cable } 2}\left\langle\left|S_{21, s}\right|^{2}\right\rangle} .
$$

$\eta_{1}$ can be obtained using (4) and (5). Thus the proposed method is mathematically derived. Compared with the method in the IEC standard [27] (Section 2.1), this method does not need SG (but VNA instead); the radiation efficiency of antennas and the cable loss do not need to be measured separately.

2.3. Method Using VNA and One Antenna. Now we are going to introduce a virtual antenna to derive the chamber transfer function of the RC using only one antenna, as shown in Figure 3. The virtual antenna is assumed to be exactly the same as antenna 2; this makes (2) become

$$
T_{2 v}=\frac{\left\langle\left|S_{21, s}\right|^{2}\right\rangle}{\left(1-\left|\left\langle S_{22}\right\rangle\right|^{2}\right)^{2} \eta_{2}^{2}} .
$$

If the chamber is well-stirred, $e_{b}=2[30,31]$, and we have

$$
\frac{\left\langle\left|S_{22, s}\right|^{2}\right\rangle}{\left\langle\left|S_{21, s}\right|^{2}\right\rangle}=2 \text {. }
$$

From (8) and (9) the chamber transfer function is only related to the parameters of antenna 2 :

$$
T_{2 v}=\frac{\left\langle\left|S_{22, s}\right|^{2}\right\rangle / 2}{\left(1-\left|\left\langle S_{22}\right\rangle\right|^{2}\right)^{2} \eta_{2}^{2}} .
$$

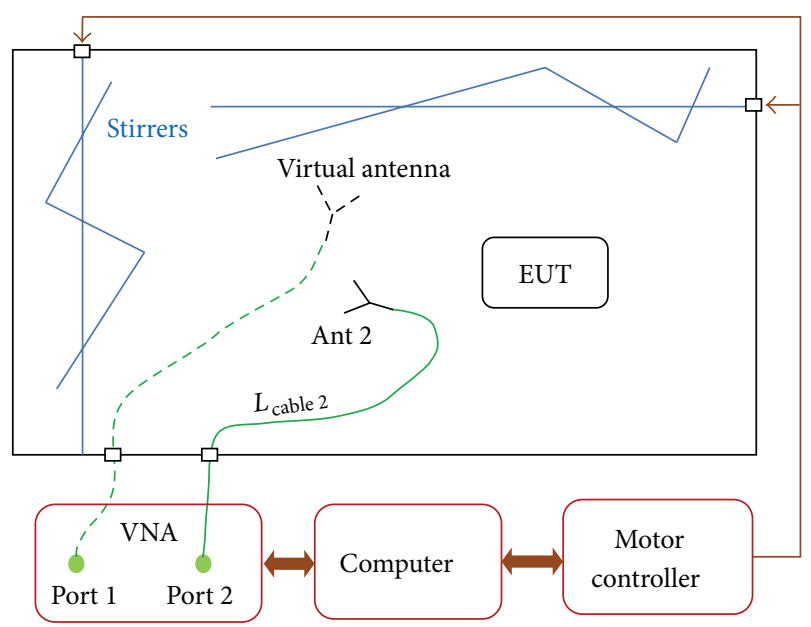

FIgURE 3: The RE measurement system with VNA and one antenna.

Let $T_{2 v}=T_{2 \mathrm{EUT}}$, and the radiated power from the EUT $\left(P_{\text {radeUT }}\right)$ can be solved as (only antenna 2 is used)

$$
P_{\text {radeUt }}=\frac{P_{\text {SAEUT }}\left(1-\left|\left\langle S_{22}\right\rangle\right|^{2}\right) \eta_{2}}{L_{\text {cable } 2}\left\langle\left|S_{22, s}\right|^{2}\right\rangle / 2} .
$$

If only antenna 1 is used, similarly we have

$$
P_{\text {radeUt }}=\frac{P_{\text {SAEUT }}\left(1-\left|\left\langle S_{11}\right\rangle\right|^{2}\right) \eta_{1}}{L_{\text {cable } 1}\left\langle\left|S_{11, s}\right|^{2}\right\rangle / 2} .
$$

Thus the proposed method with VNA and only one antenna is mathematically derived. Compared with the method in Section 2.2, only one antenna is needed.

Note that the measurement setup in Figure 3 is the same as the one-antenna method in [30] and $\eta_{1}$ and $\eta_{2}$ in (11) and (12) can be obtained using [30]

$$
\eta_{i}=\frac{1}{\left(1-\left|\left\langle S_{i i}\right\rangle\right|^{2}\right)} \sqrt{\frac{C_{\mathrm{RC}}}{2 \omega \tau_{\mathrm{RC}}}\left\langle\left|S_{i i, s}\right|^{2}\right\rangle} \quad(i=1 \text { or } 2) .
$$

In the next section, measurements are conducted to verify the proposed methods.

\section{Measurements}

The validation measurements were conducted in the $\mathrm{RC}$ at the University of Liverpool; the size of the RC is $3.6 \mathrm{~m} \times$ $4 \mathrm{~m} \times 5.8 \mathrm{~m}$. The EUT used is from Invisible Systems [32], and it is a sensor/transceiver which collects and transmits the data (temperature, electric meters, etc.) wirelessly. The carrier frequency of the sensor is $869.5 \mathrm{MHz}$ which is much larger than the lowest usable frequency (LUF) of the RC $(150 \mathrm{MHz}$ in this case). The measurement system and the sensor are shown in Figures 4(a) and 4(b); the EUT and antennas are supported by empty cartons (low loss) and are positioned with no line-of-sight paths.

Three methods in Section 2 were conducted: a logperiodic antenna (LPDA, Rohde \& Schwarz HL223) was used 


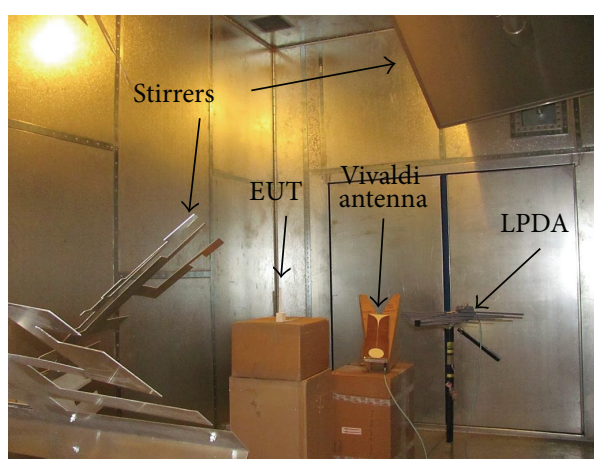

(a)

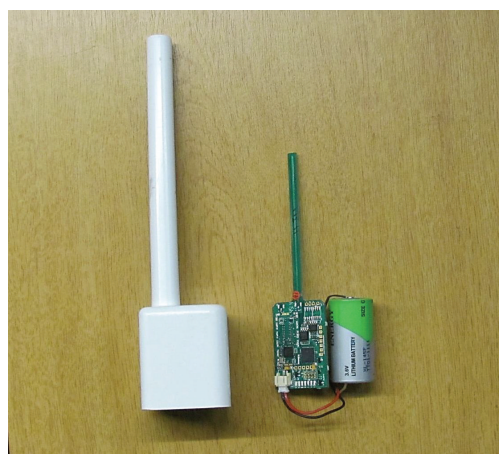

(b)

Figure 4: (a) The measurement system in RC; (b) the plastic enclosure and the sensor (EUT).

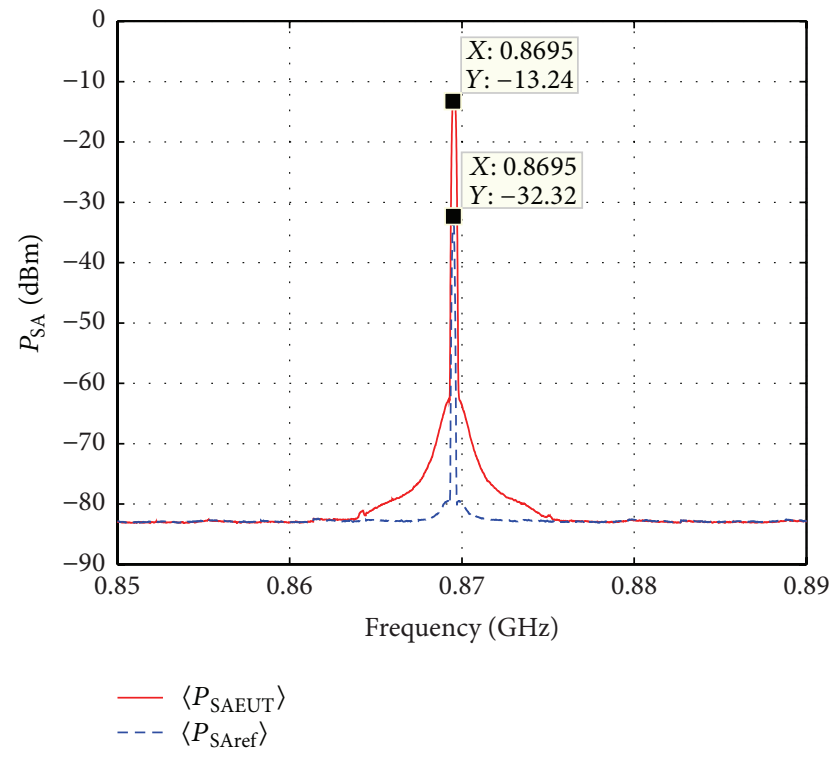

FIGURE 5: Measured averaged SA readings.

as antenna 2; a homemade wideband Vivaldi antenna was used as antenna 1 . To protect the $\mathrm{SA}$, a $10 \mathrm{~dB}$ attenuator was connected to antenna 2 to attenuate the received signal, the reference level of SG was set as $0 \mathrm{dBm}\left(P_{\text {ref }}=1 \mathrm{~mW}\right.$ at $869.5 \mathrm{MHz}$ ), 360 stirrer locations with a step size of 1 degree were used, and $S$-parameters with 10,001 points were collected in the range of $0.5 \mathrm{GHz} \sim 1.5 \mathrm{GHz}$ using a VNA. The reason why so many $S$-parameters points were collected is that we need to perform the IFFT using the $S$-parameters to obtain accurate $\tau_{\mathrm{RC}}$ [22]. The frequency stirring with 100 sample points (10 MHz bandwidth) was used to provide an averaged result for each frequency; this makes $360 \times 100=36000$ samples at each frequency.

The averaged SA readings of EUT and reference source are shown in Figure 5. The peak values are $-13.24 \mathrm{dBm}$ and $-32.32 \mathrm{dBm}$, respectively. The measured chamber decay time is extracted and shown in Figure 6 using the least square method [33]. The reflection coefficients of antennas were measured using the complex averaged $S$-parameters at

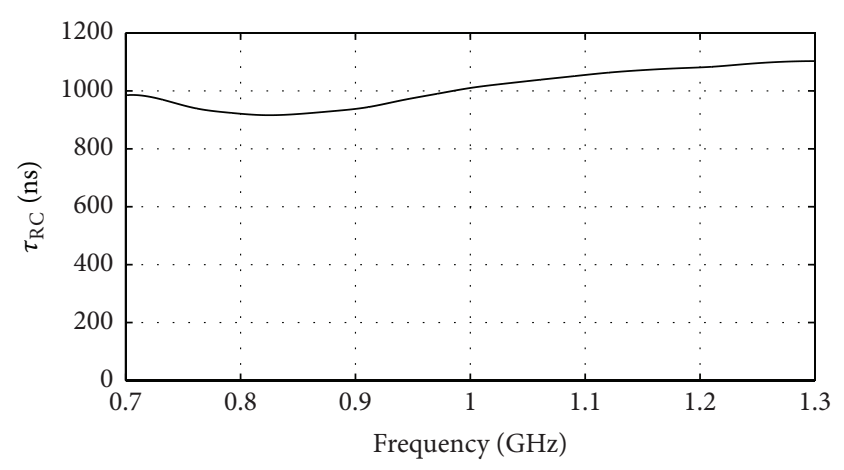

FIgURE 6: Measured chamber decay time $\tau_{\text {RC }}$.

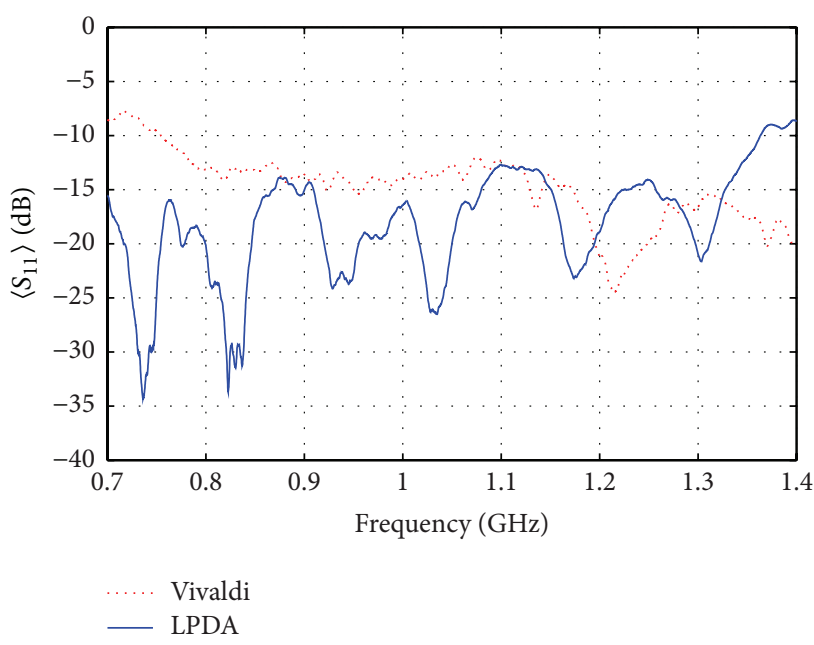

Figure 7: Measured reflection coefficients of antennas.

each frequency [34] (Figure 7). The radiation efficiency of antennas using two- and one-antenna methods is given in Figures 8 and 9 . The chamber transfer functions are obtained using (2) or (10). For one antenna method, it can be obtained from either antenna 1 or antenna 2. The results are shown in Figure 10; as can be seen, they are in a very good agreement.

The measured radiated power using three methods (1), (7), and (11)/(12) is summarized in Table 1 , and either antenna 
TABLE 1: Comparison of measured radiated power using three methods.

\begin{tabular}{lc}
\hline Method $^{*}$ & Measured radiated power $P_{\text {radeUt }}$ \\
\hline 2Ant + SG + SA (IEC standard) & $17.4 \mathrm{dBm}$ \\
2Ant + VNA + SA & $17.2 \mathrm{dBm}$ \\
1 Ant + VNA + SA (Vivaldi) & $17.2 \mathrm{dBm}$ \\
1 Ant + VNA + SA (LPDA) & $17.2 \mathrm{dBm}$ \\
\hline
\end{tabular}

${ }^{*} 2 \mathrm{Ant}+\mathrm{SG}+\mathrm{SA}$ means using 2 antennas, a signal generator and a spectrum analyzer.

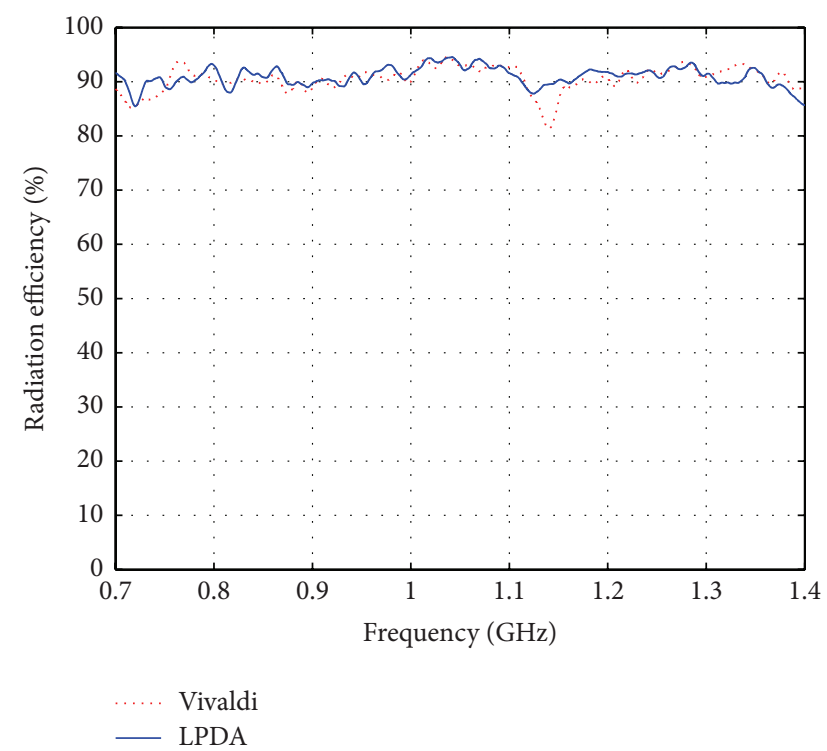

FIGURE 8: Measured radiation efficiency of antennas using twoantenna method.

1 or antenna 2 can be used in the third method (1Ant + VNA $+\mathrm{SA})$. As expected, a very good agreement is obtained; the difference is quite small $(0.2 \mathrm{~dB})$.

\section{Discussions and Conclusions}

Two new alternative methods have been proposed in this paper to measure the RE of EUT. In the IEC standard method, the loss of the cable and the performance of the antenna need to be known in advance. Also SG and two antennas are required. It has been shown that the SG is not necessary in the proposed methods; instead VNA can be used to complete the measurement and only one antenna is enough, which simplifies the measurement system without losing accuracy. This method requires minimum instruments in the RE measurement in an RC. In this paper, a two-inone instrument was used (including both a VNA and a SA). The one- and/or two-antenna method were incorporated into the RE measurement. The radiation efficiency of antennas, reflection coefficients, and the chamber transfer function were measured simultaneously.

Compared with the method in $[28,29]$, there is no need to measure the received power in the time domain, and thus no

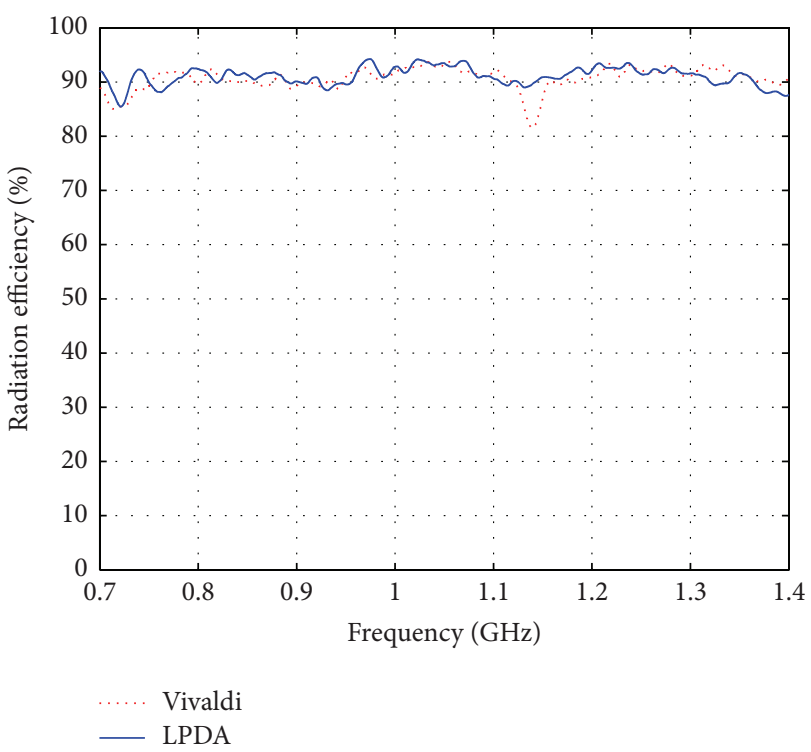

FIGURE 9: Measured radiation efficiency of antennas using oneantenna method.

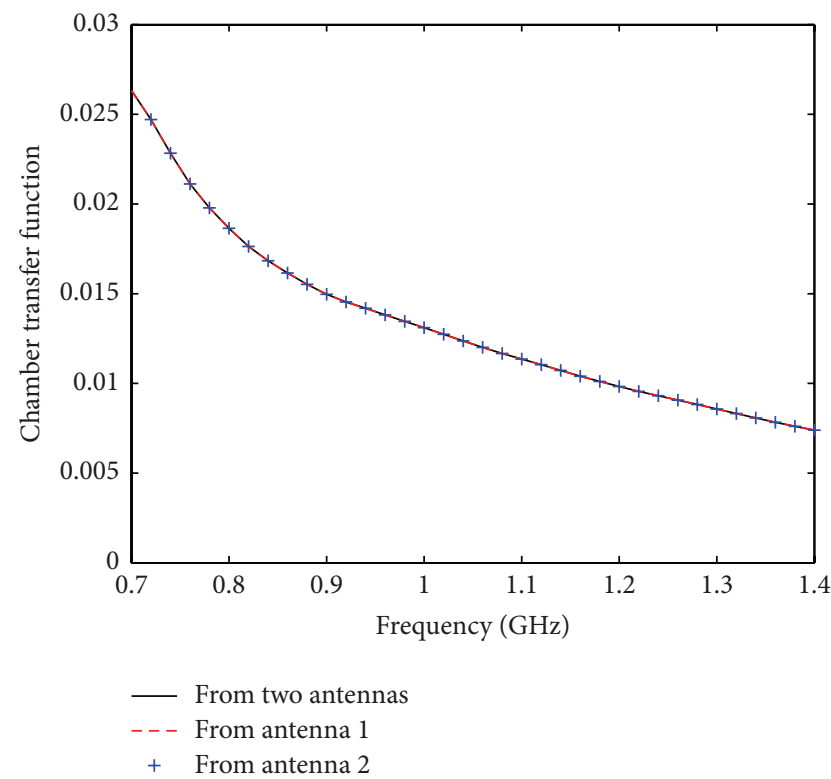

Figure 10: Measured chamber transfer function.

synchronization issue is involved. The chamber decay time is obtained from the IFFT of $S$-parameters.

There is a $0.2 \mathrm{~dB}$ difference in Table 1; if we examine the full procedure carefully, the error may come from two sources:

(1) The measurement of cable loss: in the RC, we had a relatively long cable (around 5 meters); when measuring the loss of the cable using a VNA, the cable was bent and the route might not be exactly the same as in the RE measurement; thus the cable loss may vary.

(2) The difference in the collected sample number: in the IEC standard method, no frequency stir is used, and 
the averaged received power is obtained from 360 samples (mechanical stir), while using the VNA, the sample number is 35,900 (mechanical stir + frequency stir). It is also possible to calibrate the RC in a certain frequency band using a SG, but it takes more time compared with the use of a VNA.

There are issues to be treated carefully when using the proposed methods:

(1) The proposed methods are based on the one- and/or two-antenna method. The preconditions of these methods need to be satisfied; this has been discussed in [30]. The chamber must be well-stirred and the loss of the chamber (including the EUT) is larger than the loss of antennas. However, typical values of the radiation efficiency can also be used if a higher uncertainty is acceptable (75\% for LPDA and $90 \%$ for horn antenna [27]).

(2) The transmitting time of the EUT should be much larger than the chamber decay time; in this paper, the sensor (EUT) was reprogrammed for the measurement to keep radiating. If the EUT did not radiate continuously, it might not be easy to capture the signal using a SA, or the sweep time of the SA should be set very long.

Future work may include simplifying the measurement methods/procedures in the RC by using more information from the measured data.

\section{Conflict of Interests}

The authors declare that there is no conflict of interests regarding the publication of this paper.

\section{Acknowledgments}

This work was supported in part by the Centre for Global EcoInnovation with Project no. 179 and Invisible Systems Ltd.

\section{References}

[1] J. Anguera, A. Andújar, M.-C. Huynh, C. Orlenius, C. Picher, and C. Puente, "Advances in antenna technology for wireless handheld devices," International Journal of Antennas and Propagation, vol. 2013, Article ID 838364, 25 pages, 2013.

[2] X. Chen and J. Yang, "Investigation of the effect of noise correlations on diversity gains and capacities of multiport antennas using reverberation chamber," International Journal of Antennas and Propagation, vol.2012, Article ID 486730, 9 pages, 2012.

[3] J. D. Sánchez-Heredia, P. Hallbjörner, J. F. Valenzuela-Valdés, T. Bolin, and A. M. Martínez-González, "Differences in user influence on MIMO handset antenna performance in reverberation chamber," International Journal of Antennas and Propagation, vol. 2012, Article ID 826425, 5 pages, 2012.

[4] A. Diallo, C. Luxey, P. Le Thuc, R. Staraj, and G. Kossiavas, "Diversity performance of multiantenna systems for UMTS cellular phones in different propagation environments," International Journal of Antennas and Propagation, vol. 2008, Article ID 836050, 10 pages, 2008.

[5] C. Lemoine, E. Amador, P. Besnier, J.-M. Floc'H, and A. Laisne, "Antenna directivity measurement in reverberation chamber from Rician K-factor estimation," IEEE Transactions on Antennas and Propagation, vol. 61, no. 10, pp. 5307-5310, 2013.

[6] M. Á. García-Fernández, D. Carsenat, and C. Decroze, "Antenna gain and radiation pattern measurements in reverberation chamber using Doppler effect," IEEE Transactions on Antennas and Propagation, vol. 62, no. 10, pp. 5389-5394, 2014.

[7] V. Fiumara, A. Fusco, V. Matta, and I. M. Pinto, "Free-space antenna field/pattern retrieval in reverberation environments," IEEE Antennas and Wireless Propagation Letters, vol. 4, no. 1, pp. 329-332, 2005

[8] U. Carlberg, P.-S. Kildal, A. Wolfgang, O. Sotoudeh, and C. Orlenius, "Calculated and measured absorption cross sections of lossy objects in reverberation chamber," IEEE Transactions on Electromagnetic Compatibility, vol. 46, no. 2, pp. 146-154, 2004.

[9] G. Gradoni, D. Micheli, F. Moglie, and V. Mariani Primiani, "Absorbing cross section in reverberation chamber: experimental and numerical results," Progress In Electromagnetics Research $B$, vol. 45, pp. 187-202, 2012.

[10] E. Amador, M. I. Andries, C. Lemoine, and P. Besnier, "Absorbing material characterization in a reverberation chamber," in Proceedings of the 10th International Symposium on Electromagnetic Compatibility (EMC Europe '11), pp. 117-122, IEEE, York, UK, September 2011.

[11] P. Hallbjörner, U. Carlberg, K. Madsén, and J. Andersson, "Extracting electrical material parameters of electrically large dielectric objects from reverberation chamber measurements of absorption cross section," IEEE Transactions on Electromagnetic Compatibility, vol. 47, no. 2, pp. 291-303, 2005.

[12] C. L. Holloway, D. A. Hill, J. Ladbury, G. Koepke, and R. Garzia, "Shielding effectiveness measurements of materials using nested reverberation chambers," IEEE Transactions on Electromagnetic Compatibility, vol. 45, no. 2, pp. 350-356, 2003.

[13] C. L. Holloway, D. A. Hill, M. Sandroni et al., "Use of reverberation chambers to determine the shielding effectiveness of physically small, electrically large enclosures and cavities," IEEE Transactions on Electromagnetic Compatibility, vol. 50, no. 4, pp. 770-782, 2008.

[14] A. Gifuni and M. Migliaccio, "Use of nested reverberating chambers to measure shielding effectiveness of nonreciprocal samples taking into account multiple interactions," IEEE Transactions on Electromagnetic Compatibility, vol. 50, no. 4, pp. 783786, 2008.

[15] R. Armstrong, A. Marvin, and J. Dawson, "An experimental investigation of the use of Q-factor to determine the shielding effectiveness of electrically large equipment enclosures with apertures," in Proceedings of the 10th International Symposium on Electromagnetic Compatibility (EMC Europe '11), pp. 148-152, IEEE, York, UK, September 2011.

[16] A. Gifuni, A. Sorrentino, A. Fanti et al., "On the evaluation of the shielding effectiveness of electrically large enclosure," Advanced Electromagnetics, vol. 1, no. 1, pp. 84-91, 2012.

[17] C. L. Holloway, D. A. Hill, J. M. Ladbury, P. F. Wilson, G. Koepke, and J. Coder, "On the use of reverberation chambers to simulate a Rician radio environment for the testing of wireless devices," IEEE Transactions on Antennas and Propagation, vol. 54, no. 11, pp. 3167-3177, 2006. 
[18] C. Lemoine, E. Amador, and P. Besnier, "On the $K$-factor estimation for Rician channel simulated in reverberation chamber," IEEE Transactions on Antennas and Propagation, vol. 59, no. 3, pp. 1003-1012, 2011.

[19] M. I. Andries, P. Besnier, and C. Lemoine, "Estimating K-factor and time spread parameters from a transient response of a pulse modulated sine wave in reverberation chamber," IEEE Transactions on Antennas and Propagation, vol. 61, no. 1, pp. 380-389, 2013.

[20] H. A. Shah, Wireless channel characterization of the reverberation chamber at NIST [M.S. thesis], Signals and System, Chalmers University of Technology, Gothenburg, Sweden, 2011.

[21] X. Chen, P.-S. Kildal, C. Orlenius, and J. Carlsson, "Channel sounding of loaded reverberation chamber for over-the-air testing of wireless devices: coherence bandwidth versus average mode bandwidth and delay spread," IEEE Antennas and Wireless Propagation Letters, vol. 8, pp. 678-681, 2009.

[22] E. Genender, C. L. Holloway, K. A. Remley, J. M. Ladbury, G. Koepke, and H. Garbe, "Simulating the multipath channel with a reverberation chamber: application to bit error rate measurements," IEEE Transactions on Electromagnetic Compatibility, vol. 52, no. 4, pp. 766-777, 2010.

[23] X. Chen, "Spatial correlation and ergodic capacity of MIMO channel in reverberation chamber," International Journal of Antennas and Propagation, vol. 2012, Article ID 939104, 7 pages, 2012.

[24] T. B. Hansen, "Correlation and capacity calculations with reference antennas in an isotropic environment," International Journal of Antennas and Propagation, vol. 2012, Article ID 540649, 14 pages, 2012.

[25] N. Arsalane, M. Mouhamadou, C. Decroze, D. Carsenat, M. A. Garcia-Fernandez, and T. Monedière, "3GPP channel model emulation with analysis of MIMO-LTE performances in reverberation chamber," International Journal of Antennas and Propagation, vol. 2012, Article ID 239420, 8 pages, 2012.

[26] P. Corona, G. Latmiral, E. Paolini, and L. Piccioli, "Use of a reverberating enclosure for measurements of radiated power in the microwave range," IEEE Transactions on Electromagnetic Compatibility, vol. 18, no. 2, pp. 54-59, 1976.

[27] IEC, "Electromagnetic compatibility (EMC) - part 4-21: testing and measurement techniques-reverberation chamber test methods," IEC 61000-4-21, 2011.

[28] H. G. Krauthäuser, "On the measurement of total radiated power in uncalibrated reverberation chambers," IEEE Transactions on Electromagnetic Compatibility, vol. 49, no. 2, pp. 270279, 2007.

[29] H. G. Krauthäuser and J. Nitsch, "Simplifying the measurement of total radiated power in reverberation chambers," in Proceedings of the International Conference on Electromagnetics in Advanced Applications (ICEAA '07), pp. 768-771, IEEE, Torino, Italy, September 2007.

[30] C. L. Holloway, H. A. Shah, R. J. Pirkl, W. F. Young, D. A. Hill, and J. Ladbury, "Reverberation chamber techniques for determining the radiation and total efficiency of antennas," IEEE Transactions on Antennas and Propagation, vol. 60, no. 4, pp. 1758-1770, 2012.

[31] J. M. Ladbury and D. A. Hill, "Enhanced backscatter in a reverberation chamber: inside every complex problem is a simple solution struggling to get out," in Proceedings of the IEEE International Symposium on Electromagnetic Compatibility (EMC '07), pp. 1-5, Honolulu, Hawaii, USA, July 2007.

[32] 2013, http://www.invisible-systems.com/.
[33] C. L. Holloway, H. A. Shah, R. J. Pirkl, K. A. Remley, D. A. Hill, and J. Ladbury, "Early time behavior in reverberation chambers and its effect on the relationships between coherence bandwidth, chamber decay time, RMS delay spread, and the chamber buildup time," IEEE Transactions on Electromagnetic Compatibility, vol. 54, no. 4, pp. 714-725, 2012.

[34] P.-S. Kildal, C. Carlsson, and J. Yang, "Measurement of freespace impedances of small antennas in reverberation chambers," Microwave and Optical Technology Letters, vol. 32, no. 2, pp. 112-115, 2002. 


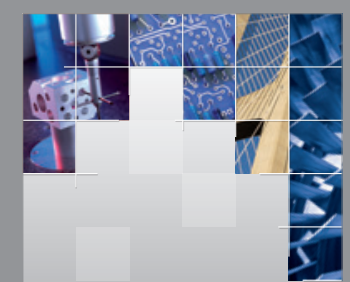

\section{Enfincering}
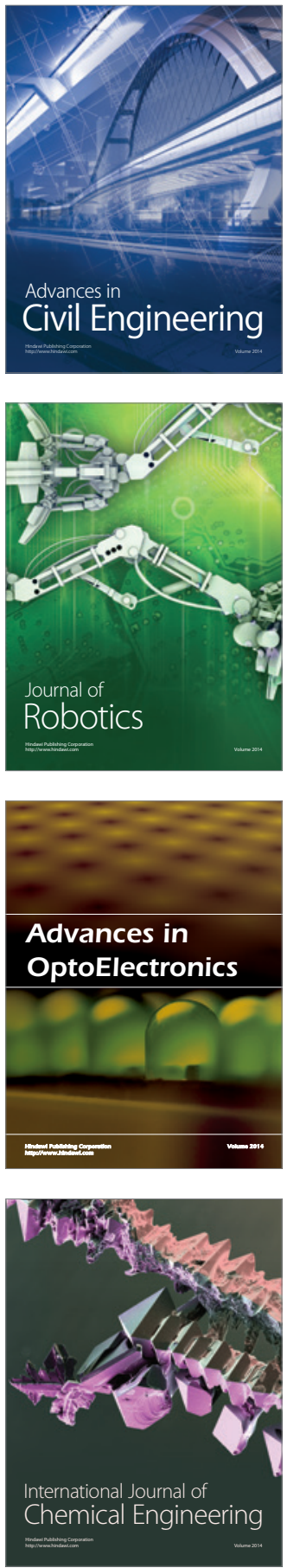

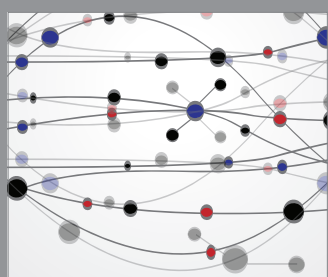

The Scientific World Journal

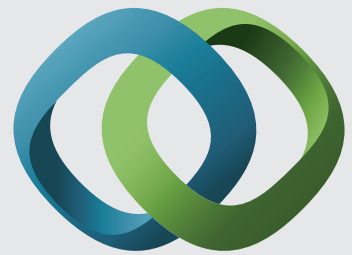

\section{Hindawi}

Submit your manuscripts at

http://www.hindawi.com
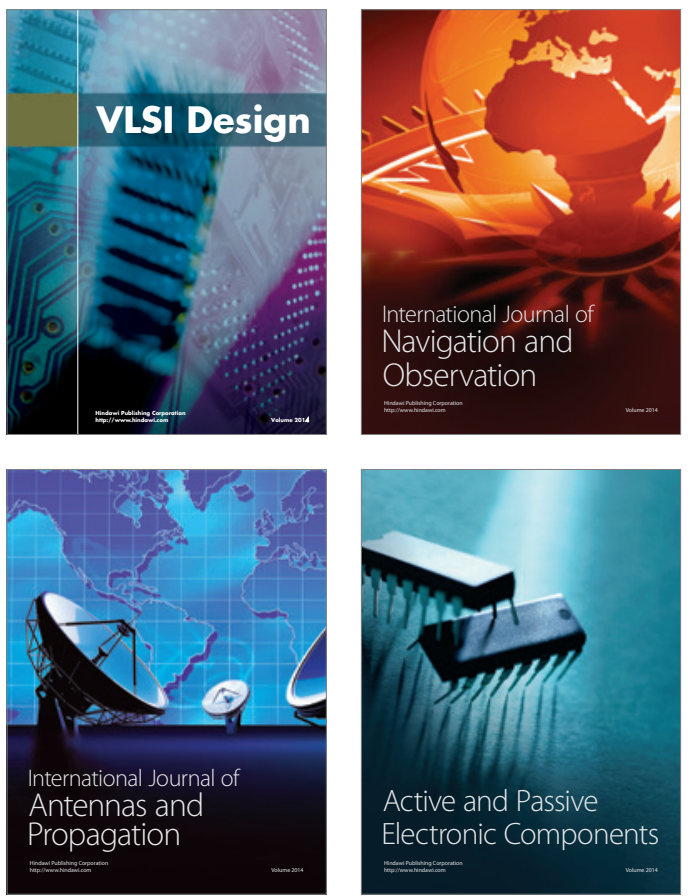
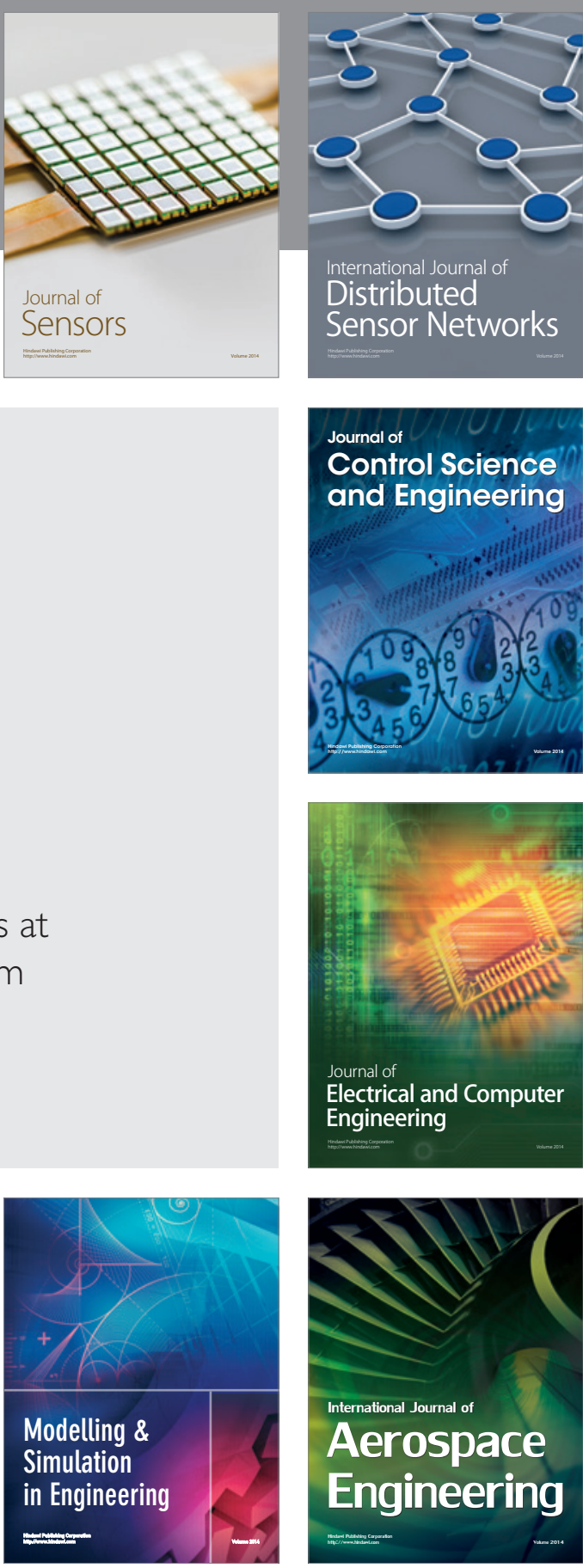

International Journal of

Distributed

Sensor Networks

Journal of

Control Science

and Engineering
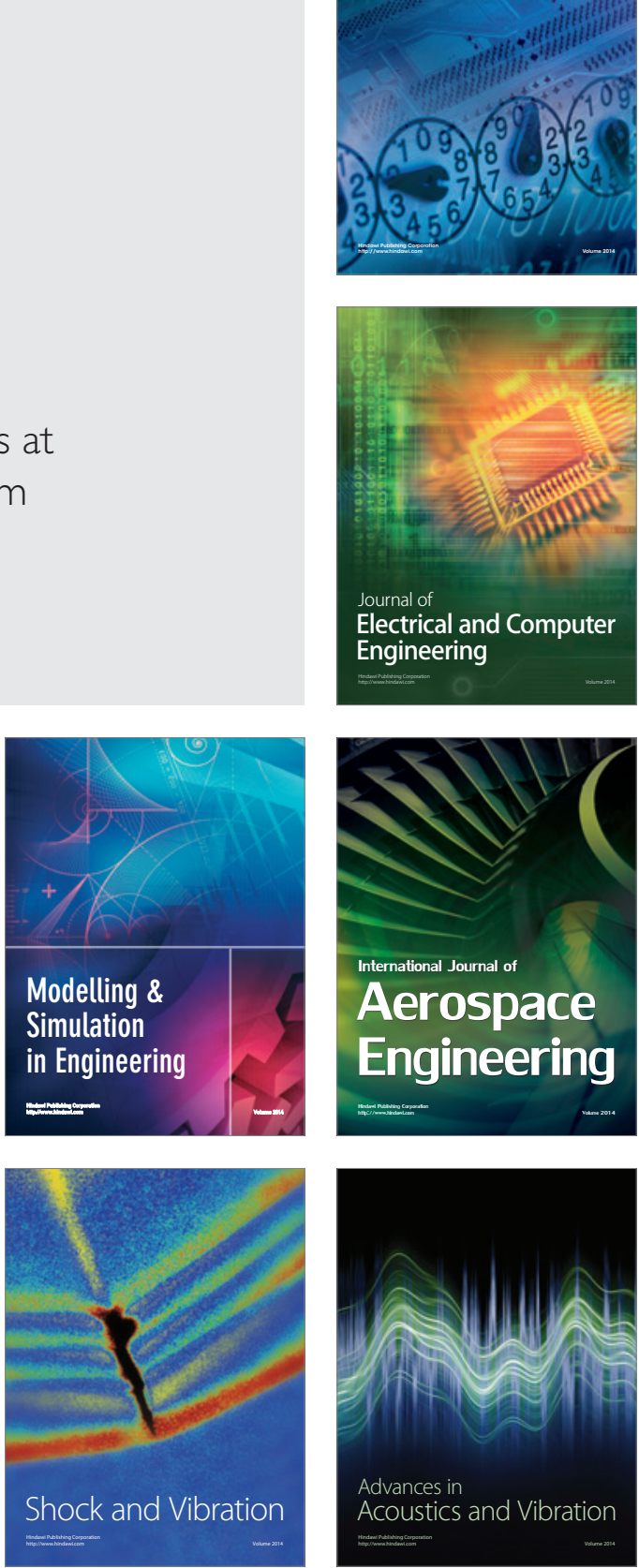